\title{
Zagreb Connection Indices of Some Classes of Networks
}

\author{
Asfand Fahad ${ }^{1}$, Adnan Aslam ${ }^{2, *}$, Muhammad Imran Qureshi ${ }^{1}$, Muhammad Kamran Jamil ${ }^{3}$, \\ Abdul Jaleel ${ }^{4}$ \\ 1 Department of Mathematics, COMSATS University Islamabad, Vehari Campus,Vehari 61100, Pakistan; \\ asfandfahad1@yahoo.com (A.F.); imranqureshi18@gmail.com (M.I.Q.); \\ 2 Department of Natural Sciences and Humanities, University of Engineering and Technology, Lahore, Pakistan; \\ adnanaslam15@yahoo.com (A.A.); \\ 3 Department of Mathematics, Riphah Institute of Computing and Applied Sciences, Lahore (RICAS), Pakistan; \\ m.kamran.sms@gmail.com (M.K.J.); \\ 4 Department of Computer Science, Rachna College of Engineering and Technology, Gujranwala, Pakistan; \\ abduljaleel@uet.edu.pk (A.J.); \\ * Correspondence: adnanaslam15@yahoo.com;
}

Scopus Author ID 55470157800

Received: 15.09.2020; Revised: 8.10.2020; Accepted: 10.10.2020; Published: 12.10.2020

\begin{abstract}
Topological indices (TIs) are numerical invariants attached to the molecular structures of the chemical compounds and are used to study their pharmacology characteristics and molecular behaviors. Several TIs presenting assistance in studying the properties of molecular structures have been defined and vastly studied. In this paper, we compute the exact values of the modified first Zagreb connection index of Silicate, Hexagonal, Oxide, and Honeycomb networks. Also, we compute the first Zagreb connection index of these networks.
\end{abstract}

Keywords: Zagreb connection index; modified first Zagreb connection number index; silicate network; hexagonal network; oxide network; honeycomb network.

(C) 2020 by the authors. This article is an open-access article distributed under the terms and conditions of the Creative Commons Attribution (CC BY) license (https://creativecommons.org/licenses/by/4.0/).

\section{Introduction}

In recent years, the computing chemistry problem has gained attention in theoretical chemistry. In chemical graph theory, we attach a function to a molecular graph that gives real number values. One of the simple functions of this type counts the number of vertices and the number of edges of the molecular graph. The important thing in assigning that function is if it can predict certain physical or chemical properties of the underlying chemical structure. These real-valued functions are known as topological indices. One of the important properties of the topological indices is that they are invariant under graph isomorphism. Several topological indices contribute to understanding the pharmaceutical aspects and properties related to the theoretical chemistry of molecular structures [1,2]. In QSPR/QSAR studies, scientists are interested to understand the topology of a chemical compound from the numerical parameters obtained from them.

We can associate a graph to molecular compounds by considering the atoms as vertices and the bond between them as edges. $\operatorname{Let} G(V(G), E(G))$ be a graph, where $V(G)$ and $E(G)$ are the set of vertices and the set of edges of the graph $G$. The number of vertices adjacent to the vertex $v$ is called the degree of the vertex $v$, and it is denoted byd $(v)$. The length of the shortest path between the verticesuand $v$ is the distance between them, and it is denoted byd $(u, v)$. For a vertex $v \in V(G), \tau_{v}$ denotes the connection number of $v$ and counts the number of vertices in 
the graph that are at a distance 2 from $v$. For more details on basic concepts related to graph theory, the readers can refer to the book [3].

A topological index assigns a numerical quantity to a graph that is invariant under graph isomorphism. The values of certain topological indices assist in predicting the various physical or chemical features associated to a chemical compound. Several topological indices have been defined and studied after the introduction of the first degree based molecular structure descriptors, by Gutman and Trinajstic [2], denoted by $M_{1}(G)$ and $M_{2}(G)$ and are defined as

$$
\begin{gathered}
M_{1}(G)=\sum_{v \in V(G)}(d(v))^{2}, \\
M_{2}(G)=\sum_{u v \in E(G)} d(u) d(v) .
\end{gathered}
$$

Using the connection number $\tau_{v}$ of a vertex $v$, Ali et al. [4] introduced the modified first Zagreb connection index, which is denoted and defined as

$$
Z C_{1}^{*}(G)=\sum_{v \in V(G)} d(v) \tau_{v}
$$

The first Zagreb connection index is denoted by $Z C_{1}(G)$ and is defined as

$$
Z C_{1}(G)=\sum_{v \in V(G)} \tau_{v}{ }^{2}
$$

Topological indices can help us to predict different properties of the understudy molecular compound. The chemical applicability of a certain topological index is tested by checking its correlating ability for the physical/chemical properties of octane isomers. In the case of the molecular descriptor $Z C_{1}^{*}$, Akbar et al. found that the correlation coefficient for entropy and acentric factor is close to 0.892 and 0.949 . This shows that the molecular descriptor $Z C_{1}^{*}$ may be useful in QSPR/QSAR studies. Ali et al. [4] studied the mathematical properties of this index. Ye et al. [5] computed $Z C_{1}^{*}$ for several molecular structures nanotubes and regular hexagonal lattices. For history and recent results on topological indices, see [6-17, 19-26].

\section{Materials and Methods}

\subsection{Zagreb connection number index for silicate networks.}

In this section, the modified first Zagreb index and first Zagreb connection index for chain silicate networks and silicate network $S L_{n}$ is computed.

Silicon tetrahedron is a basic unit of silicate, which is formed by silicon $\mathrm{Si}^{+4}$ surrounded by four oxygen atoms $O^{-2}$. The structure can be seen in [18]. Silicate is an interesting and complicated mineral with large polymeric molecules and an extensive variety of structures. The structure of silicate is also sometimes called a corner-sharing tetrahedral. Although there is some silicate with non-tetrahedral silicon, most of them contain the tetrahedral structure.

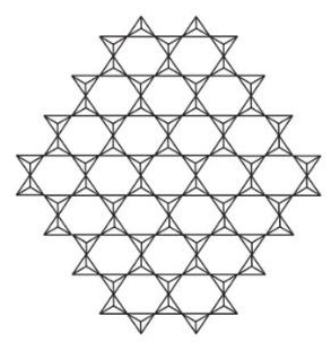

Figure 1. A Silicate network $\mathrm{SL}_{n}$ with $n=3$.

The corner atoms are called as Oxygen vertices, and central atoms are silicon vertex. The bonds between them can be considered as edges from a graphical point of view. In Figure 1, a three-dimensionalsilicate network $S L_{n}$ is shown. 
The number of vertices in $S L_{n}$ are $15 n^{2}+3 n$.

Theorem 2.1: Let $G=S L_{n}$, where $n \geq 1$. Then

i. $Z C_{1}^{*}(G)=810 n^{2}-378 n$

ii. $\quad Z C_{1}(G)=1782 n^{2}-1242 n+1188$

\section{Proof:}

i. For $G=S L_{n}$, out of a total $15 n^{2}+3 n$ vertices, $6 n^{2}+6 n$ vertices have degree $3,9 n^{2}-$ $3 n$ vertices have degree 6 . Table 1 presents the connection number for different vertices of $G$.

Table 1. Vertex partition with respect to degree and connection number for Silicate network.

\begin{tabular}{c|c|c}
$\boldsymbol{d}(\boldsymbol{v})$ & $\boldsymbol{\tau}_{\boldsymbol{v}}$ & No. of vertices \\
\hline 3 & 6 & $12 n$ \\
\hline 3 & 9 & $6 n^{2}-6 n$ \\
\hline 6 & 6 & 6 \\
\hline 6 & 9 & $12 n-12$ \\
\hline 6 & 12 & $9 n^{2}-15 n+6$
\end{tabular}

Now, by using the information computed in Table 1and the definition of $Z C_{1}^{*}$, we have,

$$
\begin{gathered}
Z C_{1}^{*}(G)=\sum_{v \in V(G)} d(v) \tau_{v} \\
=3 \times 6 \times 12 n+3 \times 9 \times\left(6 n^{2}-6 n\right)+6 \times 6 \times 6+6 \times 9 \times(12 n-12)+6 \times 12 \times\left(9 n^{2}\right. \\
-15 n+6)
\end{gathered}
$$

After simplification

$$
Z C_{1}^{*}(G)=810 n^{2}-378 n
$$

ii. By using the information from Table 1 in the definition of the $Z C_{1}$, we get the following:

$$
Z C_{1}(G)=1782 n^{2}-1242 n+1188,
$$

which completes the proof.

When in silicate minerals, the tetrahedral are arranged in a line (Linearly), then chain silicate is formed. The chain silicate of dimension $n$ is denoted by $C S_{n}$. The structure is shown in Figure 2. The total number of vertices in $C S_{n}$ are $3 n+1$.

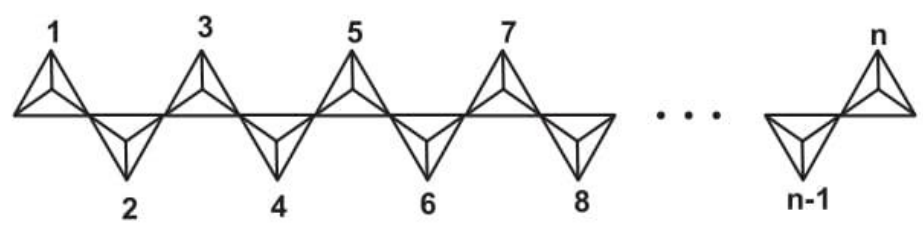

Figure 2. Chain Silicate of dimension $n$.

Theorem 2.2: Let $G=C S_{n}$, where $n \geq 1$, then

i. $Z C_{1}^{*}(G)=72 n-90$

ii. $Z C_{1}(G)=108 n-144$

\section{Proof:}

i. For $G=C S_{n}$, out of total $3 n+1$ vertices, $2 n+2$ vertices have degree 3 , and $n-1$ vertices have degree 6 . Table 2 presents the connection number for different vertices of $G$.

Table 2. Vertex partition with respect to degree and connection number for chain silicate.

\begin{tabular}{c|c|c}
$\boldsymbol{d}(\boldsymbol{v})$ & $\boldsymbol{\tau}_{\boldsymbol{v}}$ & No. of vertices \\
\hline 3 & 3 & 6 \\
\hline 3 & 6 & $2 n-4$ \\
\hline 6 & 3 & 2 \\
\hline 6 & 6 & $n-3$
\end{tabular}


Now, using the information computed in Table 2, in the definition of $Z C_{1}^{*}$, we have,

$$
\begin{gathered}
Z C_{1}^{*}(G)=\sum_{v \in V(G)} d(v) \tau_{v} \\
=3 \times 3 \times 6+3 \times 6 \times(2 n-4)+6 \times 3 \times 2+6 \times 6 \times(n-3)
\end{gathered}
$$

After simplification

$$
Z C_{1}^{*}(G)=72 n-90 .
$$

ii. By using the information from Table 2, in the definition of the $Z C_{1}$, we get the following:

$$
Z C_{1}(G)=108 n-144 \text {. }
$$

\section{Results and Discussion}

\subsection{Zagreb connection number index for some more networks.}

In this section, the modified first Zagreb index and first Zagreb connection index for Hexagonal, Oxide, and Honeycomb networks are computed.

There are a few regular planes tilling. These tilings are composed of some kind of regular polygons. In the construction of the hexagonal network, triangular tiling is being used. An $n$-dimensional hexagonal network is denoted by $H X_{n}$, here $n$ represents the number of vertices in each side of the hexagon. The Hexagonal network with $n=6$ is shown in Figure 3 .

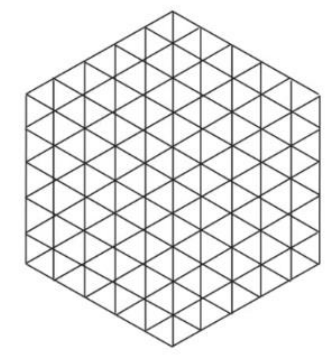

Figure 3. Hexagonal Network $\mathrm{HX}_{\mathrm{n}}$ with $n=6$.

The total number of vertices in the $n$-dimensional Hexagonal network with $n>1$ are $3 n^{2}-3 n+1$.

Theorem 3.1: If $G=H X_{n}$, where $n>1$. Then

i. $Z C_{1}^{*}(G)=216 n^{2}-588 n+354$

ii. $Z C_{1}(G)=432 n^{2}-1380 n+978$

\section{Proof:}

i. For $H X_{n}$, out of a total number of vertices $3 n^{2}-3 n+1$, the 6 vertices have degree 3 , $6 n-12$ vertices have degree 4 , and remaining vertices $3 n^{2}-9 n+7$ are of degree 6 . Table 3presents the connection number for different vertices of $G$.

Table 3. Vertex partition with respect to degree and connection number for Hexagonal Network.

\begin{tabular}{c|c|c}
$\boldsymbol{d}(\boldsymbol{v})$ & $\boldsymbol{\tau}_{\boldsymbol{v}}$ & No. of vertices \\
\hline 3 & 5 & 6 \\
\hline 4 & 6 & 12 \\
\hline 4 & 7 & $6 n-24$ \\
\hline 6 & 12 & $3 n^{2}-15 n+19$ \\
\hline 6 & 7 & 6 \\
\hline 6 & 9 & $6 n-18$
\end{tabular}

Now, using the information computed in Table 3, in the definition of $Z C_{1}^{*}$, we have, 


$$
Z C_{1}^{*}(G)=\sum_{v \in V(G)} d(v) \tau_{v}
$$

$=3 \times 5 \times 6+4 \times 6 \times 12+4 \times 7 \times(6 n-24)+6 \times 12 \times\left(3 n^{2}-15 n+19\right)+6 \times 7 \times 6$

$$
+6 \times 9 \times(6 n-18)
$$

After simplification

$$
Z C_{1}^{*}(G)=216 n^{2}-588 n+354
$$

ii. By using the information from Table 3, in the definition of the $Z C_{1}$, we get the following:

$$
Z C_{1}(G)=432 n^{2}-1380 n+978
$$

If the silicon vertices are deleted from the silicate network, the new network obtained is called an oxide network. An $n$-dimensional Oxide network is denoted by $O X_{n}$. The Figure 4 shows the 5-dimensional Oxide network $O X_{5}$.

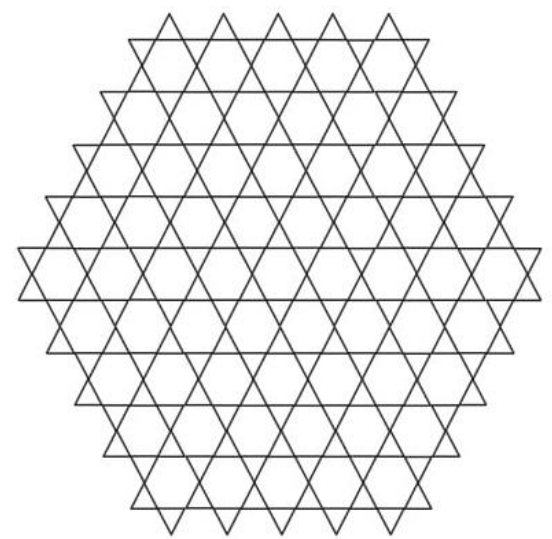

Figure 4. Oxide network $\mathrm{OX}_{5}$.

The total number of vertices in $O X_{n}$ are $9 n^{2}+3 n$.

Theorem 3.2: If $G=O X_{n}$, where $n \geq 1$, then

i. $Z C_{1}^{*}(G)=288 n^{2}-144 n$

ii. $Z C_{1}(G)=576 n^{2}-432 n+48$

\section{Proof:}

i. For $O X_{n}$, out of the total number of vertices $9 n^{2}+3 n$, the $6 n$ vertices have degree 2, $9 n^{2}-3 n$ vertices have degree 4. Table 4 presents the connection number for different vertices of $G$.

Table 4. Vertex partition with respect to degree and connection number for Oxide network.

\begin{tabular}{c|c|c}
$\boldsymbol{d}(\boldsymbol{v})$ & $\boldsymbol{\tau}_{\boldsymbol{v}}$ & No. of vertices \\
\hline 2 & 4 & $6 n$ \\
\hline 4 & 4 & 6 \\
\hline 4 & 6 & $12 n-12$ \\
\hline 4 & 8 & $9 n^{2}-15 n+6$
\end{tabular}

Now, using the information computed in Table 4, in the definition of $Z C_{1}^{*}$, we have,

$$
Z C_{1}^{*}(G)=\sum_{v \in V(G)} d(v) \tau_{v}
$$

$=2 \times 4 \times 6 n+4 \times 4 \times 6+4 \times 6 \times(12 n-12)+4 \times 8 \times\left(9 n^{2}-15 n+6\right)$

After simplification

$$
Z C_{1}^{*}(G)=288 n^{2}-144 n
$$

ii. By using the information from Table 4, in the definition of the $Z C_{1}$, we get the following: 


$$
Z C_{1}(G)=576 n^{2}-432 n+48
$$

If we recursively use Hexagonal tiling in a pattern, the honeycomb network is formed. The $n$-dimensional honeycomb network is represented by $H C_{n}$. Here $n$ represents the number of hexagons between the central and boundary hexagon. $H C_{n}$ is formed by adding a layer of hexagons around $H_{n-1}$. In Figure 5, the 4-dimensional honeycomb network is shown.

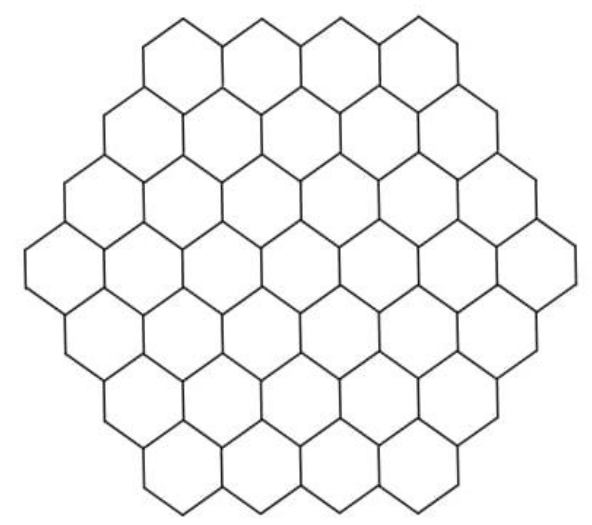

Figure 5. 4-dimensional honeycomb.

The total number of vertices in $H C_{n}$ are $6 n^{2}$.

Theorem 3.3: If $G=H C_{n}$, where $n \geq 1$, then

i. $Z C_{1}^{*}(G)=108 n^{2}-96 n+12$.

ii. $Z C_{1}(G)=216 n^{2}-240 n+36$.

\section{Proof:}

i. For $H C_{n}$, out of the total number of vertices $6 n^{2}$, the $6 n$ vertices have degree $2,6 n^{2}-6 n$ vertices have degree 3 . Table 5 presents the connection number for different vertices of $G$.

Table 5. Vertex partition with respect to degree and connection number for honeycomb.

\begin{tabular}{c|c|c}
$\boldsymbol{d}(\boldsymbol{v})$ & $\boldsymbol{\tau}_{\boldsymbol{v}}$ & No. of vertices \\
\hline 2 & 3 & 12 \\
\hline 2 & 4 & $6 n-12$ \\
\hline 3 & 4 & $6 n-6$ \\
\hline 3 & 6 & $6 n^{2}-12 n+6$
\end{tabular}

Now, using the information computed in Table 5 in the definition of $Z C_{1}^{*}$, we have,

$$
Z C_{1}^{*}(G)=\sum_{v \in V(G)} d(v) \tau_{v}
$$

$=2 \times 3 \times 12+2 \times 4 \times(6 n-12)+3 \times 4 \times(6 n-6)+3 \times 6 \times\left(6 n^{2}-12 n+6\right)$.

After simplification

$$
Z C_{1}^{*}(G)=108 n^{2}-96 n+12
$$

ii. By using the information from Table 5 in the definition of the $Z C_{1}$, we get the following:

$$
Z C_{1}(G)=216 n^{2}-240 n+36
$$

\section{Conclusions}

In this paper, we computed the exact values of the first Zagreb connection number index of Silicate, Hexagonal, Oxide, and Honeycomb networks. Moreover, the first Zagreb connection index is computed for the same networks. 


\section{Funding}

This research received no external funding.

\section{Acknowledgments}

All the authors are thankful to their respective institutes.

\section{Conflicts of Interest}

The authors declare no conflict of interest.

\section{References}

1. Gutman, I.; Polansky, O.E. Mathematical Concepts in organic chemistry.Berlin: Springer. 1986; https://doi.org/10.1007/978-3-642-70982-1.

2. Gutman, I.; Trinajstic, N. Graph theory and molecular orbitals. Total $\varphi$-electron energy of alternant hydrocarbons.Chemical Physics Letters 1972, 17, 535-538, https://doi.org/10.1016/0009-2614(72)85099-1.

3. Rosen, K.H.; Krithivasan, K. Discrete mathematics and its applications: with combinatorics and graph theory. Tata McGraw-Hill Education, 2012.

4. Ali, A.; Trinsjstic, N. A novel/old modification of the first Zagreb index. Molecular Inforamtics 2018, 37,https://doi.org/10.1002/minf.201800008.

5. Ansheng, Y.; Muhammad Imran, Q.; Asfand, F.; Adnan, A.; Muhammad Kamran, J.; Asim, Z.; Rida, I. Zagreb Connection Number Index of Nanotubes and Regular Hexagonal Lattice. Open Chemistry 2019, 17, 75-80, https://doi.org/10.1515/chem-2019-0007.

6. Furtula, B.; Gutman, I. A forgotten topological index. Journal of Mathematical Chemistry 2015, 53, 11841190, https://doi.org/10.1007/s10910-015-0480-z.

7. Iqbal, Z.; Aslam, A.; Ishaq, M.; Gao, W. The Edge Versions of Degree-Based Topological Descriptors of Dendrimers. Journal of Cluster Science 2020, 31, 445-452, https://doi.org/10.1007/s10876-019-01658-w.

8. Gao, W.; Akhter, S.; Iqbal, Z.; Qasim, M.; Aslam, A. The topological aspects of Phthalocyanines and $\begin{array}{lllll}\text { Porphyrins } & \text { Acess } & \text { 2020, } & 8,\end{array}$ https://doi.org/10.1109/ACCESS.2020.3023658.

9. Zahid, I.; Muhammad, I.; Adnan, A.; Wei, G. On eccentricity-based topological descriptors of water-soluble dendrimers. Zeitschrift für Naturforschung C 2019, 74, 25-33, https://doi.org/10.1515/znc-2018-0123.

10. Gao, W.; Aamir, M.; Iqbal, Z.; Ishaq, M.; Aslam, A. On Irregularity Measures of Some Dendrimers Structures. Mathematics 2019, 7, https://doi.org/10.3390/math7030271.

11. Iqbal, Z.; Aslam, A.; Ishaq, M.; Aamir, M. Characteristic study of irregularity measures of some nanotubes. Canadian Journal of Physics 2019, 97, 1125-1132, https://doi.org/10.1139/cjp-2018-0619.

12. Aslam, A.; Guirao, J.; Ahmad, S.; Gao, W. Topological Indices of the Line Graph of Subdivision Graph of Complete Bipartite Graphs. Applied Mathematics \& Information Sciences 2017, 11, 1631-1636.

13. Ali, H.; Binyamin, M.A.; Shafiq, M.K.; Gao, W. On the Degree-Based Topological Indices of Some Derived Networks. Mathematics 2019, 7, https://doi.org/10.3390/math7070612.

14. Wei, C.-C.; Ali, H.; Binyamin, M.A.; Naeem, M.N.; Liu, J.-B. Computing Degree Based Topological Properties of Third Type of Hex-Derived Networks. Mathematics 2019, 7, https://doi.org/10.3390/math7040368.

15. Luo, L.; Dehgardi, N.; Fahad, A. Lower Bounds on the Entire Zagreb Indices of Trees. Discrete Dynamics in Nature and Society 2020, 2020, https://doi.org/10.1155/2020/8616725.

16. Zhao, D.; Iqbal, Z.; Irfan, R.; Chaudhry, M.A.; Ishaq, M.; Jamil, M.K.; Fahad, A. Comparison of Irregularity Indices of Several Dendrimers Structures. Processes 2019, 7, https://doi.org/10.3390/pr7100662.

17. Gao, W.; Siddiqui, M.K.; Imran, M.; Jamil, M.K.; Farahani, M.R. Forgotten topological index of chemical structure in drugs. Saudi Pharmaceutical Journal 2016, 24, 258-264, https://doi.org/10.1016/j.jsps.2016.04.012.

18. Hayat, S.; Imran, M. Computation of topological indices of certain networks. Applied Mathematics and Computation 2014, 240, 213-228, https://doi.org/10.1016/j.amc.2014.04.091.

19. Goubko, M. Minimizing degree-based topological indices for trees with given number of pendent vertices.MATCH Commun. Math. Comput. Chem. 2014, 71, 33-46.

20. Gutman, I.; Das, K.C. The first zagreb index 30 years after.MATCH Commun. Math. Comput. Chem. 2004, 50, 83-92.

21. Gutman, I.; Jamil, M.K.; Akhter, N. Graphs with fixed number of pendent vertices and minimal first Zagreb index. Transactions on Combinatorics 2015, 4, 43-48, https://doi.org/10.22108/toc.2015.6029. 
22. Husin, M.N.; Hasni, R.; Arif, N.E.; Imran, M. On Topological Indices of Certain Families of Nanostar Dendrimers. Molecules 2016, 21, https://doi.org/10.3390/molecules21070821

23. Munir, M.; Nazeer, W.; Kang, S.M.; Qureshi, M.I.; Nizami, A.R.; Kwun, Y.C. Some Invariants of Jahangir Graphs. Symmetry 2017, 9, https://doi.org/10.3390/sym9010017.

24. Gao, W.; Siddiqui, M.K.; Naeem, M.; Rehman, N.A. Topological Characterization of Carbon Graphite and Crystal Cubic Carbon Structures. Molecules 2017, 22, https://doi.org/10.3390/molecules22091496.

25. Zhang, X.; Raza, A.;Fahad, A.; Jamil, M. K.; Chaudhry, M. A.; Iqbal, Z.; On Face index of Silicon Carbides, Discrete Dynamics in nature and Society, 2020, 6048438, https://doi.org/10.1155/2020/6048438.

26. Ye, A.; Javed, A.; Jamil, M.K.; Sattar, K.A.; Aslam, A.; Iqbal, Z.; Fahad, A.; On Computation of Face Index of Certain Nanotubes, Discrete Dynamics in nature and Society, 2020, 3468426, https://doi.org/10.1155/2020/3468426. 